\title{
Cost-effective Spatial Super-channel Allocation in Flex-Grid/MCF Optical Core Networks
}

\author{
Rubén Rumipamba-Zambrano, Jordi Perelló, Joan M. Gené, and Salvatore Spadaro
}

Advanced Broadband Communications Center (CCABA), Universitat Politècnica de Catalunya (UPC), Jordi Girona 1-3, 08034 Barcelona Spain, e-mail: rrumipam@ac.upc.edu

\begin{abstract}
Space Division Multiplexing (SDM) is a key technology to cope with the bandwidth limitations of single mode fibers. Multi-Core Fibers (MCFs) are considered as a promising candidate technology to implement SDM, due to their low inter-core crosstalk (ICXT), experimentally proven in laboratory prototypes. Among the different channel allocation options making use of the newly enabled space dimension, the so-called spatial super-channel (Spa-SCh) is the most likely solution to be implemented, given the inherent cost reduction of the jointswitching operation (i.e., jointly switching a spectrum portion in all MCF cores at once). This work targets the cost-effective Spa-SCh allocation over MCF-enabled Flex-Grid optical core networks. To this goal, state-of-the-art 22-core MCFs are assumed, although the proposed solutions are applicable to any MCF type. In particular, we propose and evaluate a partial-core assignment as a cost-effective strategy to improve spectrum utilization and save Capital Expenditure (CapEx) costs by minimizing the number of optical transceivers used per Spa-SCh. Numerical results reveal that reductions up to $44 \%$ and $33 \%$ in the number of active transceivers in the network can be obtained in national- and continentalwide backbone networks, respectively, without affecting the network Grade-ofService (GoS), measured in terms of Bandwidth Blocking Probability (BBP). To evaluate the impact of the ICXT, we also compare the performance of the MCF scenarios under study against equivalent Multi-Fiber (MF) ones. From the obtained results, ICXT in MCF scenarios requires the utilization of less efficient modulation formats, which reduces the admissible offered network load by up to $17 \%$ for a $1 \%$ BBP target. Furthermore, this lower spectral efficiency also demands an increase of the symbol rate per sub-channel up to a $26 \%$, a key indicator of the modulator electronic complexity.
\end{abstract}

\section{INTRODUCTION}

Space Division Multiplexing (SDM) technology arises as the "next-frontier" of fiber optics to scale up the capacity of current optical fiber systems [1]. There exist different technological SDM solutions. The straightforward one consists in upgrading the currently deployed telecom operators' technology to convert it into a Multi-Fiber (MF) infrastructure, where every link bundles several single-mode fibers. However, as in the case of Wavelength-Division Multiplexing (WDM), parallelization is a must for SDM to become economically attractive, so novel fiber designs are required [2]. A technological solution to this goal can rely on a single fiber core, large enough to transmit multiple guided modes, thus having a Multi-Mode Fiber (MMF). If the number of modes is limited to only a few, these fiber technologies are typically 
referred to as Few-Mode Fibers (FMFs). Another possibility to realize SDM is to employ fibers with several single-mode cores, called Multi-Core Fibers (MCFs). If these cores carry a few modes each, such fibers are referred to as Few-Mode Multi-Core Fibers (FM-MCFs).

These novel fibers introduce a new impairment to deal with. Namely, the coupling between modes or cores has to be considered in order to determine if equalization based on end-to-end Multiple-Input and Multiple-Output (MIMO) processing is necessary. In this regard, MCFs with several single-mode cores are particularly attractive, given their extremely low inter-core crosstalk (ICXT) values [3]-[5], alleviating the need for MIMO equalization. To further reduce ICXT, heterogeneous MCFs (cores with different characteristics) have been designed, which may be required for long-haul communications with core counts higher than 30 [6].

Over the last years, Flex-Grid has come to the fore to replace traditional WDM, given its superior flexibility to make the most of the available fiber spectral resources. Such is the case that its standardization is ongoing within the ITU-T [7]. Flex-Grid technology enables transmissions at ultra-high bit-rates by concatenating multiple adjacent flexible sub-channels (Sb-Chs), thus forming a super-channel, which are supported by software-defined optical bandwidth variable transponders (BVTXPs). In a Flex-Grid/MCF network scenario like the one considered in this work, MCFs open another degree of flexibility for super-channel configuration, namely, the spatial domain.

Several super-channel allocation flavors are possible in Flex-Grid/MCF networks, depending on the arrangement of the Sb-Chs across both spectral and spatial domains, as envisioned in [8]. For example, all Sb-Chs of the super-channel can be contiguously allocated over the same core of a MCF, forming a spectral super-channel (Spe-SCh). Conversely, they can be arranged across the spatial domain, over the same spectrum portion in all cores of a MCF, forming in this way a spatial super-channel (Spa-SCh). The combination of spectral and spatial super-channels (S2SChs) could also be possible, but predictably at expenses of higher complexity and cost, as innovative multi-dimensional nodes to switch both dimensions independently would be required [8]. The results reported in [9] show that Spe-SChs may yield better performance than Spa-SChs in terms of Bandwidth Blocking Probability (BBP), as in Spe-SChs the Guard-Bands (GBs) between Sb-Chs can be eliminated by employing Nyquist WDM [10]. However, it is also shown that Spa-SChs are potentially much less expensive in terms of hardware components, permitting to switch one spectrum portion across all cores at once, which is called jointswitching (JoS) technique [11]. The spatial dimension with JoS is considered as fixed (i.e., all cores are treated as a single entity).

Previous works $[9,11]$ asses the performance of Spa-SChs using a $50-\mathrm{GHz}$ WDM grid in MF scenarios. However, the number of spatial resources (spatial channels) to be allocated is given in advance by the traffic demand or can easily be computed taking into account a fixed transmission baud-rate and the selected modulation format. Therefore, all Spa-SChs occupy a fixed spectrum width per fiber. Our work aims to propose a cost-effective method to assign spectral and spatial resources for a given traffic demand (in b/s) in Flex-Grid/MCF networks. The proposed mechanism is evaluated by using JoS and the 22-core MCF prototype reported in [13]. In particular, when JoS is applied to Flex-Grid/MCF networks, the minimum spectral 
resources requirements per Spa-SCh are obtained when the entire spatial dimension (all cores of the MCFs) is exploited. As will be highlighted in the next sections, this can lead to spectral underutilization if such spectral requirements do not match any multiple of the frequency slot width. Our study analyzes if the spectral requirements of this latter approach can be maintained (ensuring identical network BBP performance), while minimizing (adjusting) the number of spatial channels (i.e., cores) required to support the Spa-SChs. In fact, this has the potentiality to translate into a reduction in the number of transceivers, which can further contribute to save network Capital Expenditure (CapEx) costs paving the economic viability of the Spa-SCh solution. To this end, the main aspects to be considered are: the spectral grid, the spectral continuity and contiguity constraints of the Flex-Grid technology and how to manage the ICXT for the core assignment. We shall mention here that our proposal is also valid for Flex-Grid/MF optical network scenarios, which are also evaluated later on (in subsection IV.D) to quantify the effect of the ICXT on the network performance.

The remainder of this paper is structured as follows. Section 2 describes the transmission reach estimations for MCF-enabled optical networks that we use afterwards to obtain the numerical results. Section 3 elaborates on the Spa-SCh allocation strategies. Subsection 3.1 describes the route, modulation level, core and spectrum assignment heuristic for Spa-SCh allocation. Subsection 3.2 presents a straightforward full-core assignment strategy, whereas subsection 3.3 proposes our novel cost-effective strategy, called partial-core assignment. Section 4 presents the obtained numerical results, which are organized in four subsections. Subsection 4 describes the scenario's details and assumptions. Next, subsection 4.2 describes the procedure that we follow to decide on the proper GB width value in the Spa-SCh configuration. Then, a performance comparison between the full-core and partial-core SpaSCh allocation strategies is presented in subsection 4.3. Last but not least, subsection 4.4 analyses the impact of ICXT on the performance of 22-core MCFs using an equivalent MF solution as a benchmark. Finally, section 5 draws up the main conclusions of this paper and envisions future research lines.

\section{TRANSMISSION REACH ESTIMATION}

An allocation strategy requires the estimation of the maximum Transmission Reach (TR). However, this is a difficult task, since it is dependent on many different variables like the type of fiber, the amplification scheme, the dispersion map, the nonlinear effects compensation capability, the modulation format, the channel spacing, etc.. Recently, the Gaussian noise (GN)-model has been presented in [14], and its reliability for nonlinear fiber propagation has been proven in most cases of interest. An enhanced version (EGN-model) has been also developed in [15] to provide even better accuracy, being the previous one an upper bound of the nonlinear interference (NLI) calculation, which leads to a slightly pessimistic reach estimation. Another particularity of the GN-model is that, given a specific modulation format and spectral efficiency, NLI is independent of the number of channels. In other words, it is independent of the symbol rate. In this paper, we assume the same scenario as "Link 1" in [14], whose details are listed as follows:

- 85-km spans of standard single-mode fiber (SSMF).

- Erbium-doped fiber amplifier (EDFA) with noise factor $\mathrm{NF}=5 \mathrm{~dB}$. 
- Polarization-multiplexed (PM) quadrature amplitude modulation (PM-QAM).

- Ideal Nyquist WDM (i.e, channel spacing equal to the symbol rate).

- Full Digital Signal Processing (DSP)-based chromatic dispersion compensation.

- No fiber nonlinearities compensation.

- No Polarization-Mode Dispersion.

- State-of-the-art soft Forward Error Correction (FEC) with 20\% overhead at pre-FEC BER of $2.7 \times 10^{-2}$.

- 3-dB Signal-to-Noise Ratio (SNR) margin from ideal performance and 1.5-dB penalty of soft FEC with respect to infinite-length codes ideal performance.

The estimated TR corresponding to each modulation format is provided in Table I. This is a pessimistic estimation for several reasons. Firstly, as mentioned previously, the GN-model is intrinsically pessimistic. Secondly, the channel spacing is set to its theoretical minimum, while in our simulations GBs are included. Thirdly, no fiber nonlinearities compensation is assumed. Lastly, no Raman amplification is employed.

TABLE I

TR in $\mathrm{km}$ given by the GN-Model [14]

\begin{tabular}{|c|c|c|c|}
\hline BPSK & QPSK & 16-QAM & 64-QAM \\
\hline$>20,000$ & 9,000 & 2,000 & 600 \\
\hline
\end{tabular}

TABLE II

XT-limited TR in km for the 22-core MCF [13]

\begin{tabular}{|c|c|c|c|}
\hline BPSK & QPSK & 16-QAM & 64-QAM \\
\hline 6,607 & 3,311 & 832 & 209 \\
\hline
\end{tabular}

To estimate the transmission reach in a MCF scenario, ICXT has to be taken into account. In principle, Spa-SChs span over the entire spatial domain when JoS is realized, so that the maximum XT interference appears. Since estimations based on average ICXT may be way too optimistic, in this study we have considered the worst ICXT introduced by the fiber along propagation and the maximum linear in-band XT tolerated by each modulation format for a 1 dB Optical Signal-to-Noise Ratio (OSNR) penalty [16]. Under this assumption, Spa-SChs can use any core index (no core assignment restriction is necessary). The present study assumes that each network link deploys the homogeneous 22-core MCF prototype presented in [13] with a measured ICXT equal to $-56.2 \mathrm{~dB} / \mathrm{km}$ (referred to $1550 \mathrm{~nm}$ ). Hence, the maximum transmission reach values limited by ICXT in our Flex-Grid/MCF network are shown in Table II, where a 4-dB margin is considered similarly to the nonlinear propagation case.

As can be seen in Tables I and II, the transmission reach is always limited by ICXT. Even though this would not have happened if a MCF with fewer cores would have been considered, we wanted to assume in this paper long-term MCF-enabled network scenarios, where the impact of ICXT could be relevant. Heterogeneous MCFs prototypes with even higher core counts could have been considered as well, but in this study we aimed to concentrate on the limits of the homogeneous MCFs.

\section{SPATIAL SUPER-CHANNEL ALLOCATION}

This section elaborates on different strategies that can be adopted to allocate a Spa-SCh in a 
Flex-Grid/MCF dynamic optical network, highlighting their benefits and drawbacks. To this end, a greedy heuristic to serve the incoming Spa-SCh requests is firstly presented. In this heuristic, a Spa-SCh configuration procedure is included, which will be particularized in subsections III.A and III.B, so as to realize the full-core and partial-core Spa-SCh allocation strategies introduced before. Since JoS switches one spectral portion across all cores at once, the resource allocation problem is equivalent to Flex-Grid over a single spatial channel. Therefore, the allocation procedure is reduced to a Route, Modulation level and Spectrum Assignment (RMSA) problem. To ease the comprehension of the RMSA heuristic and Spa-SCh allocation strategies, Table III details the nomenclature used in their description. As suggested in [7] and [17], we call Frequency Slot Units (FSUs) to the spectrum blocks (of $12.5 \mathrm{GHz}$ in our work) into which the available spectrum in MCF cores is discretized; moreover, we also call Frequency Slot (FS) to a frequency range built from the concatenation of a number of contiguous FSUs.

TABLE III

NOMENCLATURE USED IN THE DESCRIPTION OF THE RMSA HEURISTIC, FCA AND PCA

\begin{tabular}{|c|c|}
\hline Symbol & Definition \\
\hline Spa-SCh & Spatial super-channel \\
\hline$S b^{-} C h$ & $\begin{array}{l}\text { Sub-channel. One Spa-SCh is composed by two or more } \\
\text { Sb-Chs, one per core carrying a fraction of the demand } \\
\text { requested bit-rate. }\end{array}$ \\
\hline$\Delta_{S b}$ & Sb-Ch spectral width (occupied bandwidth per $\mathrm{Sb}-\mathrm{Ch}$ ) \\
\hline$d$ & $\begin{array}{l}\text { Incoming bidirectional demand to be served by setting up } \\
\text { a Spa-SCh }\end{array}$ \\
\hline$s_{d}, t_{d}$ & Source and destination nodes of demand $d$ \\
\hline$r_{d}$ & Total requested bit-rate by demand $d$ \\
\hline$G(V, E)$ & $\begin{array}{l}\text { Graph representing the optical network, where } \mathrm{V} \text { is the set } \\
\text { of optical nodes (e.g., SDM-capable Reconfigurable Optical } \\
\text { Add/Drop Multiplexers (ROADMs)) and E the set of } \\
\text { bidirectional optical links (i.e., MCFs) }\end{array}$ \\
\hline$|C|$ & Number of cores of the deployed MCFs \\
\hline$F S U$ & Frequency Slot Unit (i.e., a $12.5 \mathrm{GHz}$ spectrum block) \\
\hline$F S$ & Frequency Slot (i.e., a number of contiguous FSUs) \\
\hline$\Delta_{\text {fsu }}$ & FSU width (i.e., $12.5 \mathrm{GHz}$ ) \\
\hline$K$ & $\begin{array}{l}\text { Number of candidate physical paths over which the Spa- } \\
\text { SCh may be established }\end{array}$ \\
\hline$S E$ & $\begin{array}{l}\text { Spectral efficiency in bits } / \mathrm{s} / \mathrm{Hz} \text { of the selected modulation } \\
\text { format for all Sb-Chs of the } \mathrm{Spa}-\mathrm{SCh}\end{array}$ \\
\hline$n$ & Number of bits per symbol of the modulation format \\
\hline$R_{S}^{\max }$ & Maximum baud-rate of the optical modulators \\
\hline$R_{S}$ & Operational baud-rate of the optical modulators \\
\hline$f_{c}$ & Central frequency of the Spa-SCh \\
\hline$n_{f s u}$ & Number of FSUs needed per core to allocate the Spa-SCh \\
\hline$G B$ & Guard-band spectral width \\
\hline$\Delta_{f}$ & $\begin{array}{l}\text { Bandwidth per core necessary to allocate the Spa-SCh, } \\
\text { including guard-bands }\end{array}$ \\
\hline$n_{c}$ & Number of cores used when configuring the Spa-SCh \\
\hline
\end{tabular}

\subsection{RMSA heuristic details}

The proposed RMSA heuristic is shown in Pseudo-code 1 . When a demand request $d$ arrives at 
the network, the $K$ feasible candidate shortest paths (SPs) between its source $\left(s_{d}\right)$ and destination $\left(t_{d}\right)$ nodes are computed, taking their physical length (in $\mathrm{km}$ ) as a metric. In this paper, we always assume $K=3$, which provides good enough network performance and low heuristic complexity. Even though the consideration of a larger number of (longer) candidate paths could further avoid the blocking of demand $d$, an excessive amount of resources might be required (higher number of hops traversed, utilization of a modulation format with low spectral efficiency), causing the blocking of multiple demand requests in the future.

Next, for each of the computed candidate paths we select the most efficient modulation format with a TR equal or higher than its physical length, based on the TR estimations reported in Section II. Then, we compute the minimum number of contiguous FSUs $\left(n_{f s u}\right)$ per core necessary to allocate the Spa-SCh over the currently evaluated candidate path between its $s_{d}$ and $t_{d}$ nodes. To obtain this value, we use the following expression:

$$
n_{f s u}=\left\lceil\frac{r_{d} /(|C| \cdot S E)+G B}{\Delta_{f s u}}\right\rceil
$$

where $r_{d}$ is the total requested bit-rate of demand $d,|C|$ is the number of cores in the MCFs, $\mathrm{SE}$ is the Spectral Efficiency (in bits $/ \mathrm{s} / \mathrm{Hz}$ ) of the employed modulation format, $\mathrm{GB}$ is the guardband width that should surround each Sb-Ch of the Spa-SCh, and $\Delta_{f s u}$ the FSU width (i.e., 12.5 $\mathrm{GHz}$, as suggested by the ITU-T for Flex-Grid in [7]). Note that, by equally splitting $r_{d}$ over all available cores of the MCFs, we are indeed squeezing the spectral requirements of the Spa-SCh to the minimum ones.

The next step performed by the heuristic is to find if a FS of the quantified $n_{f s u}$ FSUs (including GBs) is available in the first core along the evaluated candidate path, ensuring the spectrum continuity (i.e., no spectrum conversion capability is assumed in the network) and contiguity constraints. The first available FS found is chosen, that is, a first-fit spectrum assignment is applied. If no available FS of $n_{f s u}$ FSUs is found along the evaluated candidate path, the heuristic moves to evaluate the following candidate path, performing the same steps again (i.e., modulation format selection, $n_{f s u}$ computation and spectrum assignment). If no FS is found on any of the $K$ candidate physical paths, the incoming demand is considered as blocked.

Assuming that a FS has been found along any of the $K$ candidate physical paths, this means that the Spa-SCh can be established. Therefore, the Spa-SCh configuration procedure can be initiated. The following two subsections introduce two different strategies to configure SpaSChs employing JoS technique, namely, the Full-Core Assignment (FCA) and Partial-Core Assignment (PCA). Both approaches contemplate three degrees of flexibility: baud-rate, modulation format and sub-channel multiplicity. Besides, they can be applied to either MF or MCF solutions and with any switching paradigm [19]; however, the present study is focused on their evaluation in a Flex-Grid/MCF network.

\begin{tabular}{ll}
\hline Pseudo-code 1: RMCSA heuristic \\
\hline 1: & Input: \\
& $G=(V, E) / /$ Physical Network, V: Set of nodes, E: Set of links. \\
& $d / /$ Incoming demand request to be served \\
$2:$ & Begin:
\end{tabular}




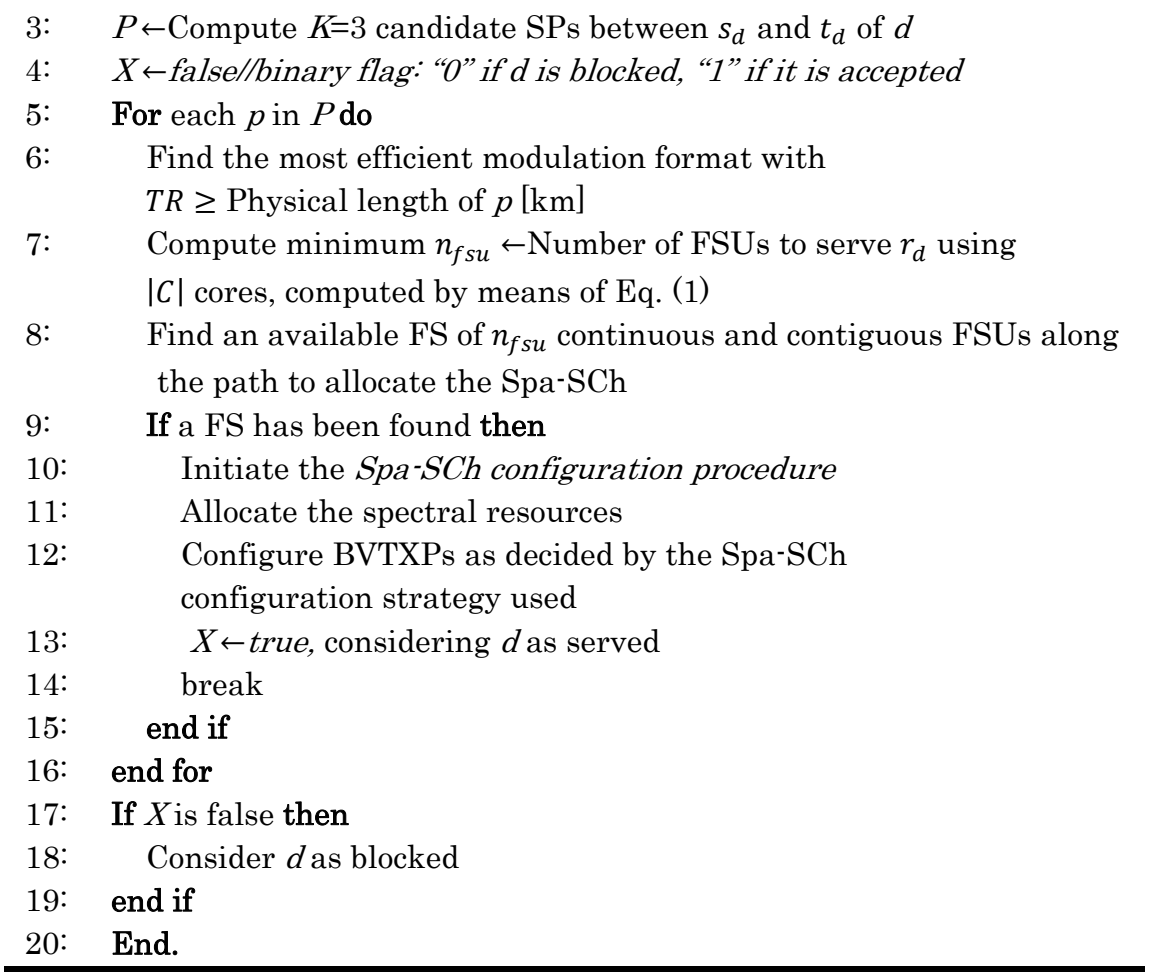

\subsection{Full Core Assignment (FCA) strategy}

This strategy is based on the decision to configure the Spa-SCh using all $|C| \mathrm{MCF}$ cores. Then, each core supports a Sb-Ch at bit-rate $r_{d} /|C|$ over the FS found, employing the previously selected modulation format. Therefore, $|C|$ transceivers are required to establish the Spa-SCh operating at $R_{S}=r_{d} /(|C| \cdot n)$ Baud. This is illustrated in Fig. 1 (a). As seen, the Spa-SCh can be generated using one optical modulator per core, plus a single laser emitting the optical carrier shared by all Sb-Chs at frequency $f_{c}$. Each of these Sb-Chs contains a fraction of the original demand volume (equal to $r_{d} /|C|$ ), split by means of a serial-to-parallel converter (S/P). Moreover, a Spa-SCh configuration requires that each $\mathrm{Sb}-\mathrm{Ch}$ has a GB (half per $\mathrm{Sb}-\mathrm{Ch}$ side, as also reflected in Fig. 1) to allow the optical filters of the Spectrum Selective Switches (SSSs) the proper capture of the incoming optical signals. All Sb-Chs of the Spa-SCh can be switched together at intermediate nodes by means of the JoS technique.

Although FCA targets the minimization of the bandwidth $\left(\Delta_{f}\right)$ required to allocate the Spa-SCh by using the whole spatial domain, it may happen that a significant portion of spectrum still remains wasted. This can occur when the $\mathrm{Sb}$-Ch bandwidth does not tightly fit into an integer number of FSUs. To put this in numbers, let us assume in Fig. 1(a) that 22-core MCFs (from $c_{1}$ to $c_{22}$ in Fig. 1) have been deployed in the network, and that we want to serve a $1 \mathrm{~Tb} / \mathrm{s}$ incoming demand request by means of a Spa-SCh configured using the FCA strategy. Moreover, we assume that the length of the physical path allows us to modulate the Sb-Chs using PM-64QAM. If we assume $10 \mathrm{GHz}$ GBs ( $5 \mathrm{GHz}$ per side), which is a typical GB width assumed in the related literature [17], we need a FS of $2 \mathrm{FSUs}\left(\Delta_{f}=25 \mathrm{GHz}\right)$, but the spectrum occupied by the Sb-Chs $\left(\Delta_{S b}\right)$ reduces to merely $3.8 \mathrm{GHz}[1000 \mathrm{~Gb} / \mathrm{s} \div(22$ cores $\cdot 12 \mathrm{~b} / \mathrm{s} / \mathrm{Hz})]$. That is, there exists an $11.2 \mathrm{GHz}$ of spectrum gap per core (underutilized spectrum) left by FCA. 


\subsection{Partial Core Assignment (PCA) strategy}

Upon situations such as those mentioned above in the FCA strategy, it would be much more efficient to adjust the number of assigned cores to $n_{c} \in \mathbb{Z}^{+}: 1 \leq n_{c} \leq|C|$, thus increasing the core spectrum utilization, while reducing the number of transceivers to be used per Spa-SCh. That is, the $n_{f s u}$ FSUs specified in pseudo-code 1 are allocated in $n_{c}$ cores instead of allocating in the entire $|C|$ available cores (as FCA does). The JoS technique continues being applied, but we release spatial (cores) and spectral resources, yet providing the same network GoS as FCA.

In order to fill the abovementioned spectrum gaps left by FCA in each core, we find the maximum Sb-Ch spectral width $\left(\Delta_{S b}\right)$ by subtracting the GB width from $\Delta_{f}$ (max $\Delta_{S b}=n_{f s u}$. $\left.\Delta_{f s}-G B\right)$. Next, the programmable BVTXPs are configured to operate at $R_{S}=\max \Delta_{S b}$ if $R_{S} \leq R_{S}^{\max }$ (i.e., their maximum baud-rate is not exceeded). Otherwise, they are configured to operate at $R_{S}=R_{S}^{\max }$. In this way, the total number of cores used to transmit the Spa-SCh with PCA is $n_{c}=\left\lceil r_{d} /\left(R_{S} \cdot n\right)\right\rceil$. Note that the aggregate bit-rate $\left(R_{S} \cdot n \cdot n_{c}\right)$ with PCA can become slightly higher than $r_{d}$ as a result of the ceiling operation. However, the final operational baud-rate could be readjusted later to $R_{s}=r_{d} /\left(n \cdot n_{c}\right)$.
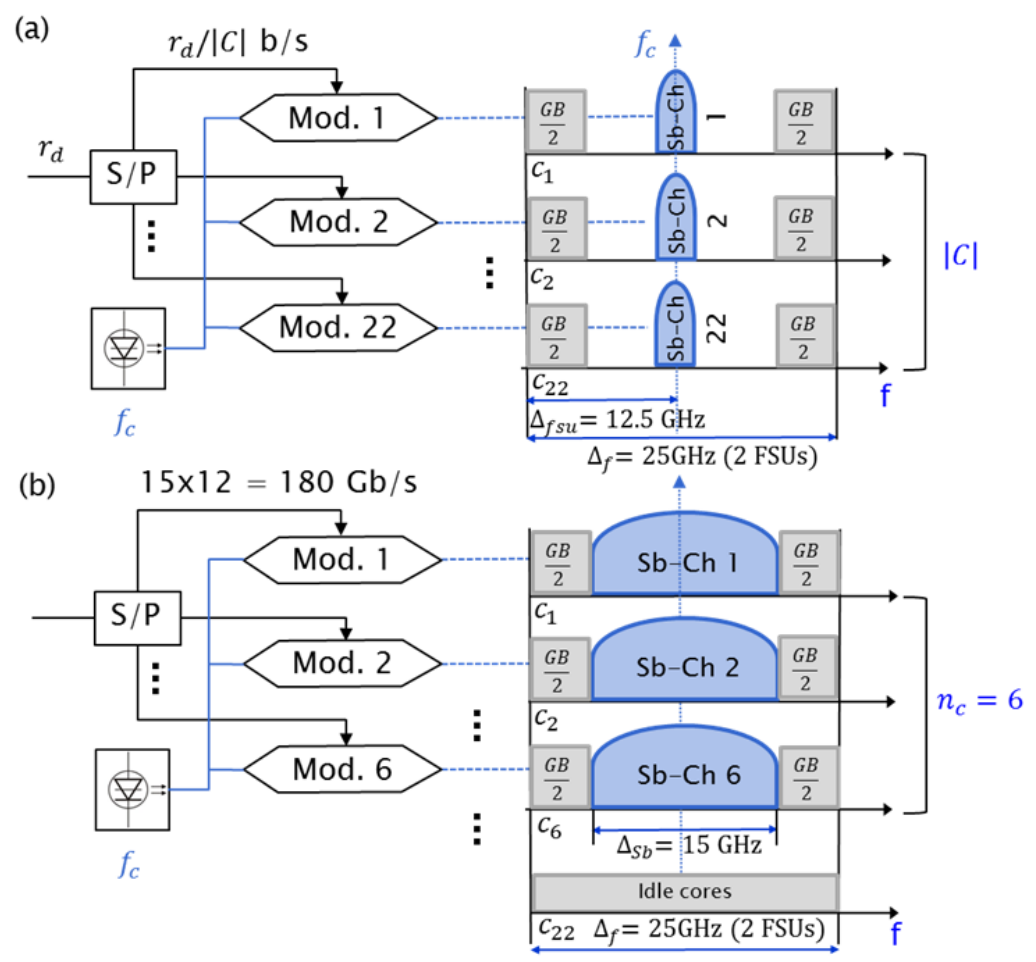

Fig. 1. Spa-SCh allocation using FCA (a) and PCA (b) strategies. 22-core MCFs are considered. There, a Spa-SCh has to be allocated to support a $1 \mathrm{~Tb} / \mathrm{s}$ demand. Optical modulators with maximum flexible baud-rate of 32 GBaud employing PM-64QAM are assumed. Guard-bands (GBs) are set to $10 \mathrm{GHz}$.

The benefits achievable by using PCA are illustrated in Fig. 1(b), where the same assumptions previously made for exemplifying the performance of FCA are considered. In contrast to FCA, with PCA we have that $\max \Delta_{S b}$ can be $15 \mathrm{GHz}(2 \cdot 12.5-10)$. Then, assuming a maximum flexible baud-rate of the transceivers equal to 32 GBaud $\left(R_{s}^{\max }=32 \mathrm{GBaud}\right), n_{c}$ can be decreased down to $6([1000 /(15 \cdot 12)])$, considering that PM-64QAM is employed as in the 
previous example. So, each core transmits $180 \mathrm{~Gb} / \mathrm{s}(15 \cdot 12)$ and the aggregate bit-rate is $1080 \mathrm{~Gb} / \mathrm{s}(15 \cdot 12 \cdot 6)$. Note that the allocated spectrum continues being the same as in the FCA strategy (i.e., 2 FSUs), ensuring identical network BBP. Nevertheless, as 6 cores are supporting the Spa-SCh, only 6 optical modulators (and consequently 6 coherent optical detectors) are required. In conclusion, adjusting the number of cores simultaneously used improves the spectral occupancy (avoiding spectrum wastage) and potentially reduces the number of transceivers to be equipped per node, which will be quantified in section IV.C.

\section{Numerical results}

\subsection{Scenario Details and Assumptions}

In order to assess the performance of FCA and PCA strategies, we consider two network topologies: the Deutsche Telekom national backbone network (DT12) and a long-haul US backbone network (US26), shown in Fig. 2 together with their main characteristics. Each network link consists of a 22-core MCF, one per direction, with a worst aggregate ICXT of -56.2 $\mathrm{dB} / \mathrm{km}$ [13]. Moreover, each MCF core has $320 \mathrm{FSUs}$ of $12.5 \mathrm{GHz}$ width available along the 4 $\mathrm{THz}$ C-Band.

A dynamic scenario is assumed, where demand requests arrive at the network one after another following a Poisson process, with a negative exponentially distributed Inter-Arrival Time (IAT). Each request asks for a bidirectional lightpath between randomly chosen source and destination nodes (uniform traffic distribution in the network) with bit-rate equal to $r_{d}$ during a certain Holding Time (HT), which also follows a negative exponential distribution. To decide on the specific $r_{d}$ value, we assume a long-term traffic profile (TP) with predominance of ultra-high bit-rate super-channel requirements. To this end, we assume that $r_{d}$ can take any of the following bit-rates with equal probability, $\operatorname{TP}=\{400,600,800,1000,1200,1400\} \mathrm{Gb} / \mathrm{s}$, resulting in an average bit-rate per demand of $900 \mathrm{~Gb} / \mathrm{s}$. To simulate different offered load (L) conditions (i.e., $L=H T / I A T$ ), we fix the mean IAT value, and modify the mean $H T$ value of the super-channels accordingly. To get statistically relevant results, we offer $2 \times 10^{5}$ demand requests per execution.

Optical signals in the Sb-Chs of the Spa-SCh are modulated considering dual-polarization phase \& quadrature modulators (PDM I/Q modulators). We consider the typical industry standard value of 32 GBaud [14], as the maximum operational baud-rate of the transceivers. Therefore, for practical purposes, transceivers can occupy up to $32 \mathrm{GHz}$ spectral width. If we consider a $\mathrm{GB}$ width from 0 to $12.5 \mathrm{GHz}$, then the maximum number of FSUs assigned per core and demand will be equal to 4 (or $\Delta_{f}=50 \mathrm{GHz}$ ). All Sb-Chs of a Spa-SCh are always transmitted using the same modulation format, which can be chosen among PM-BPSK, PM-QPSK, PM-16QAM and PM-64QAM, depending on the TR requirements. Thus, the bit-rate per Sb-Ch can be up to 64, 128, 256, $384 \mathrm{~Gb} / \mathrm{s}$, respectively. For example, employing PM-BPSK in a 22-core MCF, the maximum $r_{d}$ that can be served (transmitting one Sb-Ch per core) is $1408 \mathrm{~Gb} / \mathrm{s}$. 


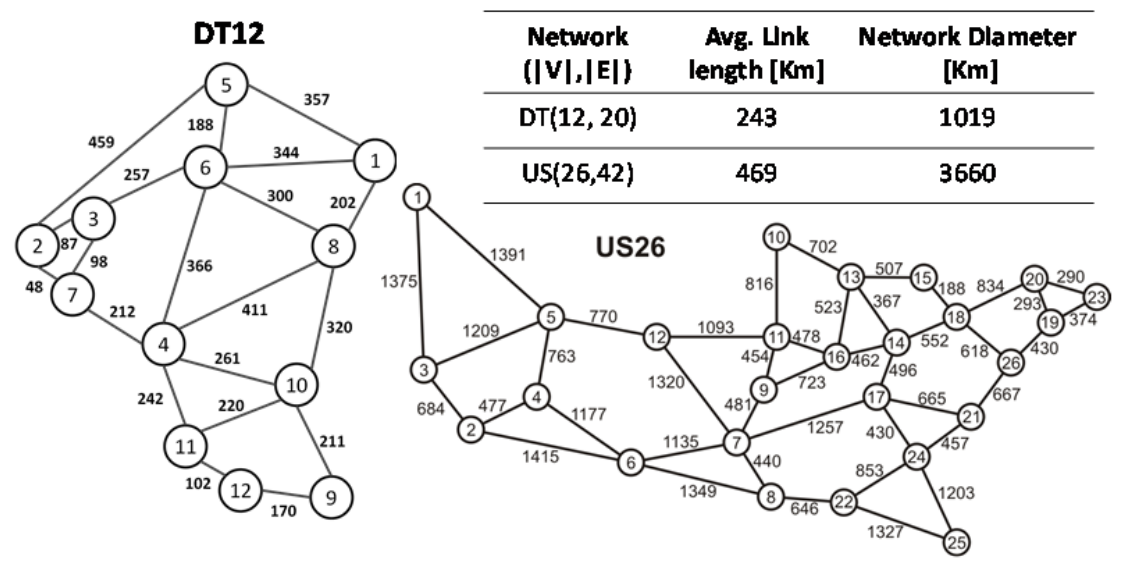

Fig. 2. Considered backbone network topologies and main characteristics.

\subsection{Guard-Band Setting}

In section II we mentioned that a drawback of Spa-SChs is the spectral waste caused by the GBs that have to surround each sub-channel. In this subsection, prior to comparing our PCA vs. the straightforward FCA strategy in detail, we aim to assess the impact of different GB values on the Flex-Grid/MCF performance, measured in terms of the experienced BBP. For this, we start from a very commonly assumed GB width of $12.5 \mathrm{GHz}[9,11]$, decreasing it until the ideal $0 \mathrm{GHz}$ value with $2.5 \mathrm{GHz}$ granularity. This should give us an insight into intermediate GB width values, adoptable by current and/or short-term SSS filter devices, while yielding increased network performance.

A key design goal of the PCA strategy is to firstly achieve the same BBP as FCA (i.e., using the same number of FSs), and then try to save transceivers by compacting the Spa-SCh bit-rate onto a fewer number of cores. This first goal is illustrated in the results of Figs. 3(a) and 3(b), where only one curve per GB width is plotted representing both FCA and PCA (identical performance in terms of $B B P$, no matter the $G B$ width value assumed). Apart from this, we can see that for a specific offered load, BBP performance depends on the combination of two factors, namely, the network size and GB. 

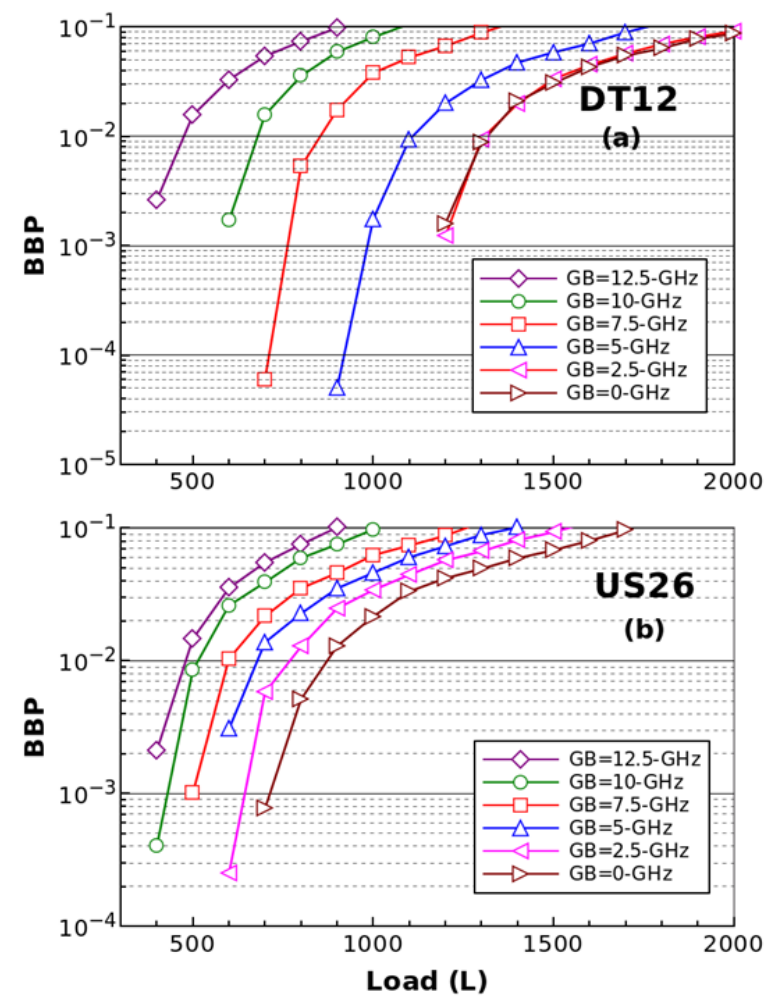

Fig. 3. BBP metric vs. offered network load in the DT12 (a) and US26 (b) network assuming 22-core MCFs per link. The performance (BBP) of both FCA and PCA strategies is identical; hence, only one curve per GB width value is depicted in each network.

In the case of DT12 network depicted in Fig. 3(a), for a target BBP $=1 \%$, every $2.5 \mathrm{GHz} G B$ width reduction generally translates into significant improvements in the admissible offered load (up to $44 \%$ increase), except for the transition from 2.5 to $0 \mathrm{GHz}$, for which the same BBP performance is observed. This can be explained as follows. Referring to Eq. (1), it may occur that even reducing the GB width, $\Delta_{f}$ remains identical, since the allocated spectrum $\Delta_{f}$ has a strong dependence on the FSU granularity $(12.5 \mathrm{GHz}$ in this work). In some particular cases, this may happen for any of the bit-rates in the traffic profile and employing any modulation format. As an example, let us consider a $1400 \mathrm{~Gb} / \mathrm{s}$ demand to be served with any of the following modulation formats: PM-QPSK, PM-16QAM, and PM-64QAM in a 22-core MCF network. According to Eq. (1) for both $2.5 \mathrm{GHz}$ and $0 \mathrm{GHz}$ the number of FSUs $\left(n_{f s u}\right)$ allocated are always 2, 1 and 1, respectively. This does not occur in the case of the US26 network (Fig. 3, b). As it can be seen, improvements in terms of extra load that can be offered for the same $\mathrm{BBP}=1 \%$ target can reach up to $20 \%$ per $2.5 \mathrm{GHz}$ GB width reduction step.

As an outcome of this subsection results, we select an adequate $G B$ width value based on two considerations: the BBP performance benefits and the technological constraints. In this sense, among the different GBs analyzed, the BBP benefits obtained with a GB equal to $7.5 \mathrm{GHz}$ are significant. Indeed, we have verified that, for a target $\mathrm{BBP}=1 \%$, reducing $\mathrm{GB}$ from 12.5 to 7.5 $\mathrm{GHz}$ allows increasing the offered load by $77 \%$ and $25 \%$ in the DT12 and US26 networks, respectively (Fig. 3). Moreover, a $7.5 \mathrm{GHz}$ GB is technologically feasible given the fine resolution of the state-of-the art of SSS filters [18]. Therefore, a GB width of $7.5 \mathrm{GHz}$ is assumed in all results presented hereafter. 


\subsection{Comparison of PCA and FCA strategies}

As explained in the previous subsection, FCA and PCA strategies yield identical BBP performance. In this subsection, we aim to evaluate network CapEx reduction that could be achieved by employing the PCA strategy through the minimization of the required number of cores/transceivers.

In a dynamic Flex-Grid/MCF network scenario as the one assumed in the present paper, every time an incoming demand $d$ is accepted, $n_{c}$ transceivers should be assigned to the programmable BVTXPs, so as to properly generate the Spa-SCh. These transceivers will then be released once the demand is torn down (its HT expires). In order to illustrate the number of transceivers that would be required in the network, we get samples of the number of active transceivers in the network $(\alpha)$ every time a Spa-SCh has been allocated. We do this in both DT12 and US26 networks with 22-core MCFs per link and under an offered load yielding a BBP of $1 \%$ (i.e., load L=850 and L=600 for DT12 and US26 networks, respectively, as shown in Fig. $3)$, aiming to evaluate a reasonable network scenario.

Fig. 4 and 5 show $\alpha$ histograms using both FCA (in blue) and PCA (in purple) strategies in the DT12 and US26 networks. They depict the percentage of samples (occurrences) where the measured $\alpha$ has fallen into each of the predefined $X$ axis intervals. Recall that we offer $2 \times 10^{5}$ demand requests per simulation execution. Although some of these requests will be blocked eventually, the low BBP in the evaluated network scenario ensures that more than $1.98 \times 10^{5}$ samples are used to generate these histograms, as larger connections (in terms of bit-rate) are more prone to blocking than smaller ones.

Looking at the obtained results, they describe a Gaussian-like probability distribution along the evaluated $\alpha$ intervals, although for PCA it is clearly shifted to the left, showing a remarkable reduction of the active transceivers. Indeed, in the DT12 network (Fig. 4), while with FCA the average $\alpha$ value is 36376 transceivers, with PCA it is reduced to 20317 transceivers (i.e., $44 \%$ reduction). Another very interesting outcome is that with PCA no occurrence falls into those $\alpha$ intervals larger than 23500, which could be taken as the peak number of active transceivers required in the network. Conversely, with FCA this peak value shifts until 40900. This also represents a reduction around 44\%. In the US26 network (Fig. 5), the average $\alpha$ value reduction achieved by PCA is around 33\% (26230 vs. 17665). In terms of peak active transceivers, a reduction around $33 \%$ is also obtained (30500 vs. 20300). 


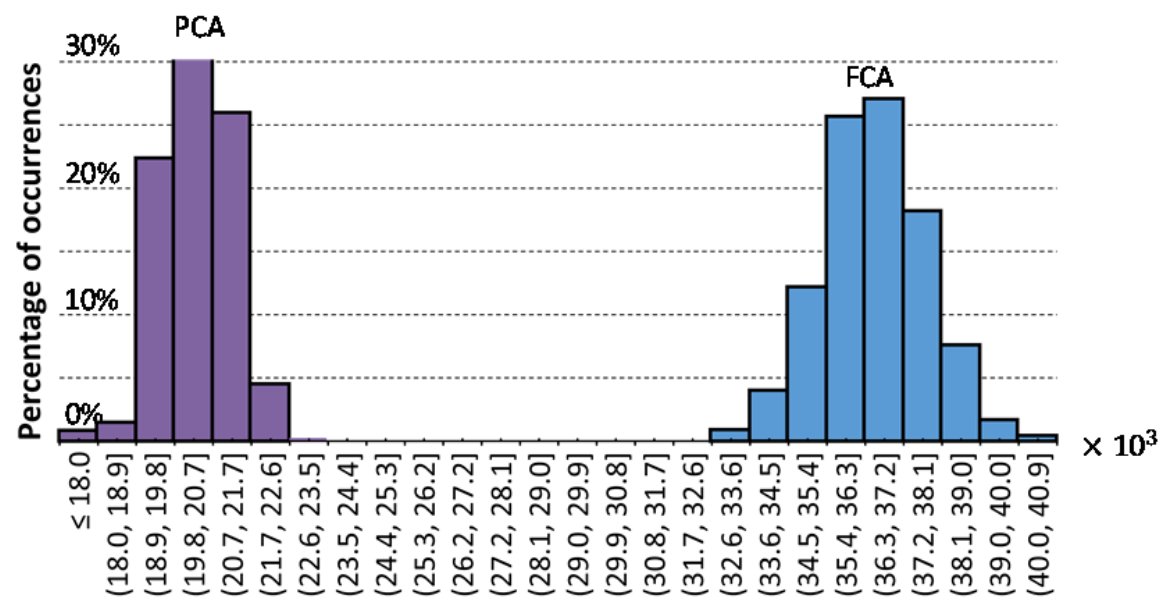

Number of Active Transceivers in the network $(\alpha)$

Fig. 4. $\alpha$ histogram in the DT12 network with 22-core MCFs using FCA (in blue) and PCA (in purple) strategies. An offered load yielding a 1\% BBP has been considered.

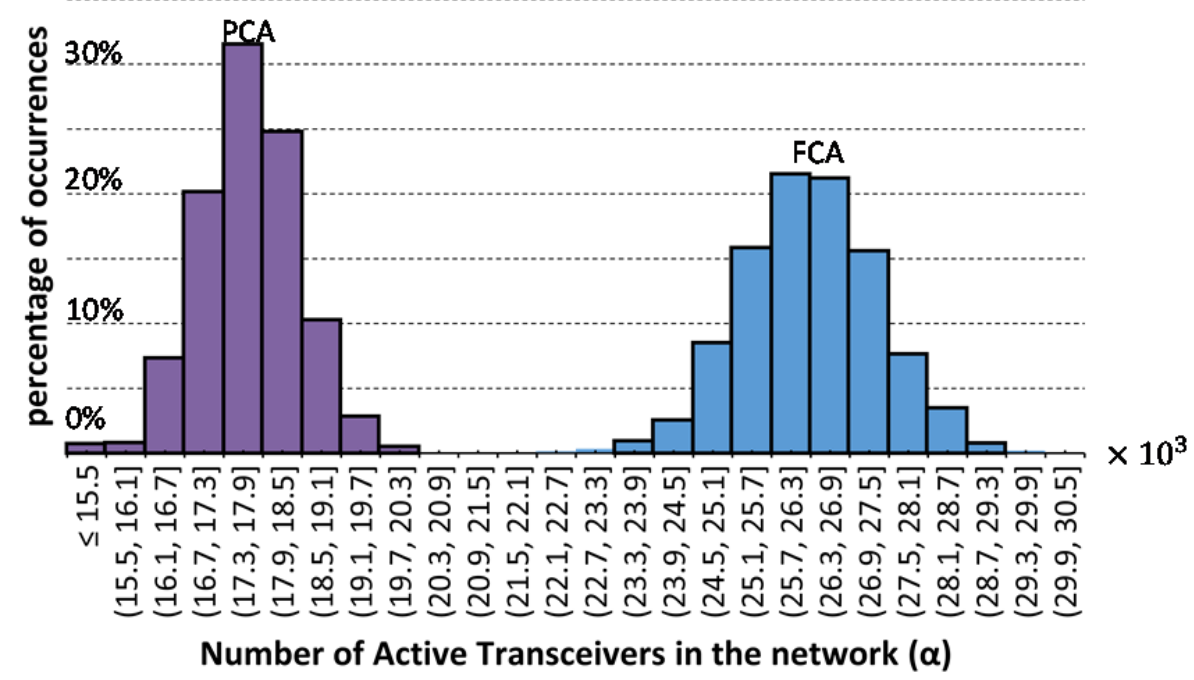

Fig. 5. $\alpha$ histogram in the US26 network with 22-core MCFs using FCA (in blue) and PCA (in purple) strategies. An offered load yielding a 1\% BBP has been considered.

To ensure that no demand request blocking happens by transceiver unavailability, the aforementioned peak active transceivers requirement should at least be equipped networkwide. In order to evaluate this behavior also at the node level, we have measured the average peak number of active transceivers per node along the whole simulation, getting again samples after each Spa-SCh allocation. We have observed that the average number of transceivers to be equipped per node using PCA is 2229 and 1084 in the DT12 and US26 networks, respectively. These requirements can be contrasted with those obtained by using FCA, which are 3913 and 1577 in both networks, respectively. Hence, the proposed PCA strategy can potentially yield substantial CapEx savings derived from the lower requirements in terms of optical transceivers to be equipped at network nodes. Of course, although out of the scope of this work, an accurate offline Flex-Grid/MCF network planning contemplating PCA vs. FCA would also be welcome to give further insight in this regard. 
Note that, in spite of the longer distances in the US26 continental backbone network, it requires a lower number of transceivers per node than the national DT12 backbone network. This means the longer distances have required to modulate the signals with less efficient modulation formats, causing to allocate wider spectrum widths (consequently higher baud rates) but transmitting in a lower number of cores $\left(n_{c}\right)$. However, the operational baud-rate of the transceivers in the US26 network will be higher. To shed light on this concern, Figs. 6(a) and $6(\mathrm{~b})$ show the transceiver operational baud-rate histograms in the DT12 and US26 networks, respectively. Again, an offered load leading to $1 \%$ BBP has been considered. We can observe that, for PCA in the DT12 network, the percentages of occurrences are almost equally concentrated in two intervals $(4,5]$ and $(17,18]$ GBaud, whilst in the US26 there is a high percentage $(73 \%)$ of the operational baud-rates corresponding to the $(17,18]$ GBaud interval plus a small one $(2.2 \%)$ of baud-rates to the $(29,30]$ and $(31,32]$ GBaud intervals. To be more concise, the mean baud-rate is 10.95 GBaud and 14.69 GBaud in the DT12 and US26 networks, respectively, which are intrinsically higher than in FCA taking into account that PCA tries to maximize the spectrum occupied per spectrum allocated. For example, when the demands require allocating 1, 2 or $3 \mathrm{FSUs}$ with a $7.5 \mathrm{GHz}$ GB, the maximum operational baud-rates are: $5,17.5$ or 30 GBaud, respectively. Note that, the low percentage of occurrences that require the highest baud-rates, 30 and 32 GBaud, in US26 could be discarded due to its high cost of generation/detection, presumably with the BBP metric being not greatly affected. Regarding the FCA strategy, we can observe that the operational baud-rates are more granular than PCA, because they have more flexibility, as FCA always employs all $|C|$ cores without considering the maximization of the spectrum occupation. The lower baud-rate requirements of FCA may look like an advantage; however, it should be seen in terms of transceiver's bandwidth utilization. In our case the nodes are equipped with 32GBaud transceivers, which are underused in the case of FCA. Such high bandwidth transceivers are a must in view of an eventual traffic demand increase.

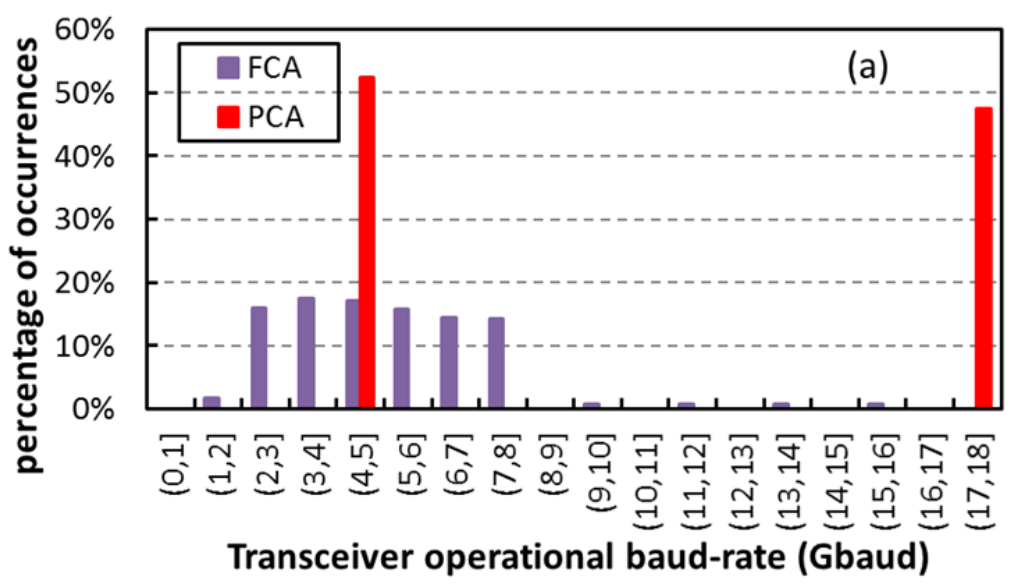




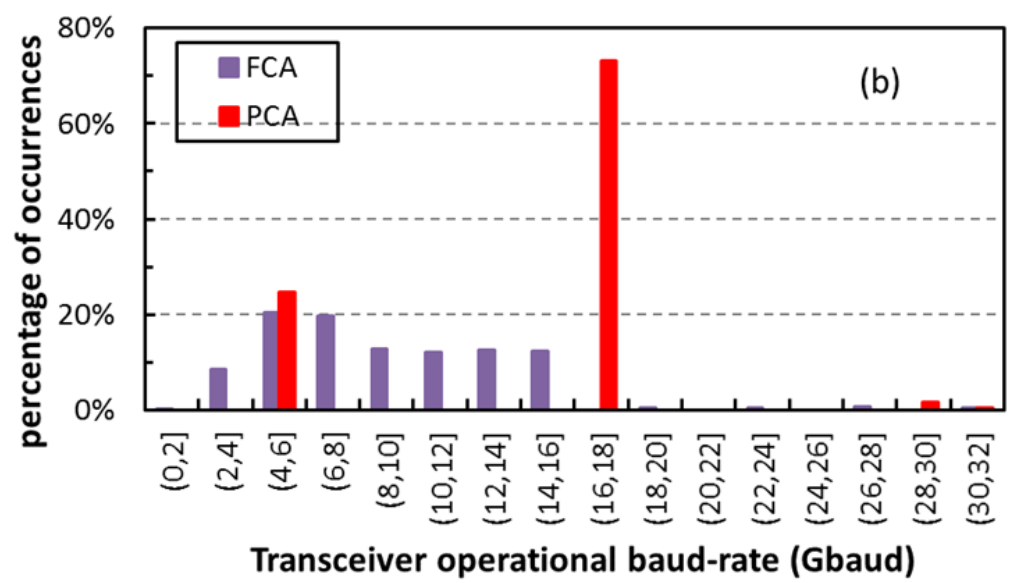

Fig. 6. Transceiver operational baud-rate histogram in the DT12 (a) and US26 (b) network. An offered load leading to BBP around 1\% has been considered.

\subsection{ICXT Impact Analysis}

To enhance the evaluation of MCFs scenarios, in this subsection we investigate its impact on the network performance. For benchmarking purposes, in this subsection a Flex-Grid/MF equivalent scenario has been also considered. For a given transmission distance, ICXT may impose the necessity of less efficient modulation formats in a MCF-enabled network compared to an equivalent MF alternative (as no coupling exists between bundles of single-core fibers). In more detail, ICXT can lead to one of the following three situations when allocating a Spa-SCh to carry an incoming demand request:

i) The number of cores per Spa-SCh $\left(n_{c}\right)$ to carry the entire $r_{d}$ is increased (compared to the MF equivalent scenario), as a lower bit-rate is transmitted per core. However, the number of required FS $\left(n_{f s u}\right)$ stays unaffected.

ii) $n_{f s u}$ is increased. However, as the Spa-SCh has allocated a wider bandwidth, a higher bitrate may be transmitted per core and, thus, $n_{c}$ can be lowered.

iii) $n_{f s u}$ is increased. This time the reduction of the $S E$ also leads to an $n_{c}$ increase (more cores are required for the Spa-SCh).

To exemplify these situations, let us assume that a $1 \mathrm{~Tb} / \mathrm{s}$ demand should be carried by means of a Spa-SCh, either over a MCF-enabled or an equivalent MF network. The first situation (i) would occur if the end-to-end path length is equal to $1000 \mathrm{~km}$. In this case, the most efficient modulation format in a MF scenario is PM-16QAM. Therefore, we have that $n_{f s u}=2$ and $n_{c}=8$ if the PCA strategy is employed. In contrast, in a 22-core MCF network, the most efficient modulation format lowers to PM-QPSK. So, we still have $n_{f s u}=2$, but $n_{c}$ raises up to 15. As for the second situation (ii), it would occur if the end-to-end path length would shorten to $400 \mathrm{~km}$. The most efficient modulation format in a MF scenario is PM-64QAM. Therefore, $n_{f s u}=1$ and $n_{c}=17$. In 22-core MCF scenario, the most efficient modulation format lowers to PM-16QAM. Thus, $n_{f s u}=2$ and $n_{c}=8$ (we have a bandwidth increment and the number of cores used is reduced). Finally, the third situation (iii) would occur if the end-to-end path would lengthen to $3500 \mathrm{~km}$. The most efficient modulation format in a MF scenario is PMQPSK, so that $n_{f s u}=2$ and $n_{c}=15$. However, in a 22-core MCF scenario, the most efficient 
modulation format lowers to PM-BPSK, resulting in $n_{f s u}=3$ and $n_{c}=17$ (i.e., an increment of both bandwidth and the number of cores used). Assuming that JoS is employed at intermediate nodes, situations ii) and iii) lead to higher BBP in the MCF-enabled network compared to the equivalent MF case, as a wider FS is necessary to allocate the same superchannel. To quantify such negative effects of ICXT, Fig. 7 depicts the network BBP vs. offered load in our Flex-Grid/MCF network scenarios describing the DT12 and US26 network topologies, together with that of an equivalent MF scenario with a bundle of 22 independent single-mode fibers per link. The proposed PCA strategy is employed in all cases. Moreover, as argued at the beginning of this section, a GB equal to $7.5 \mathrm{GHz}$ has been assumed. As can be observed in the figure, the MF clearly outperforms the MCF-enabled one. For example, for a target $\mathrm{BBP}=1 \%$, around $14 \%$ and $17 \%$ offered load reduction is evidenced in the MCF-enabled scenario versus the equivalent MF in the DT12 and US26 networks, respectively.

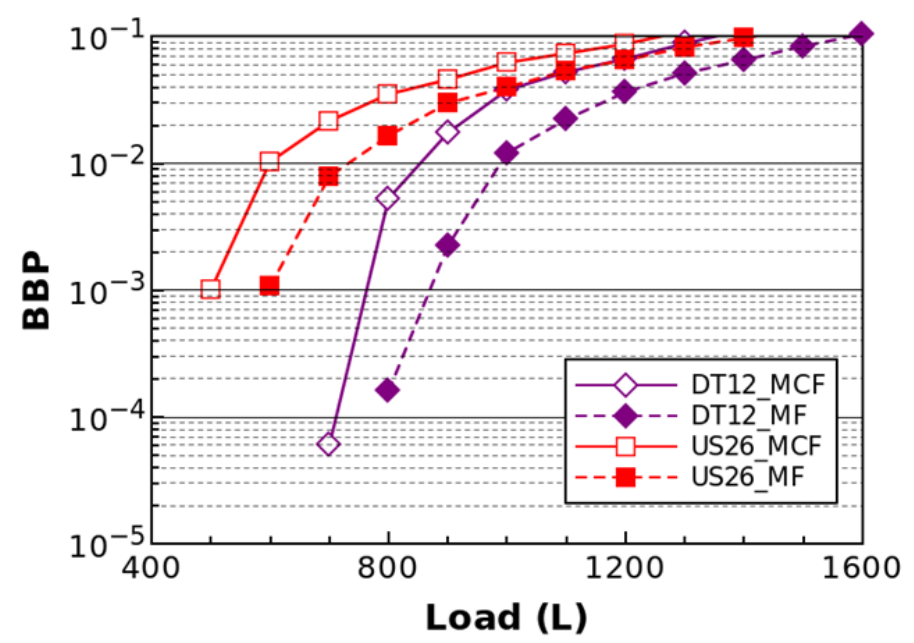

Fig. 7. BBP vs. offered load in MCF-enabled and MF DT12 and US26 network topologies. 22-core MCFs or bundles of 22 fibers per link are assumed in each case. GB=7.5 GHz.

To complement this analysis, Fig. 8 shows the distribution of the usage of the modulation formats in MCF scenarios compared to MF ones. To obtain these results, we have focused on an offered load leading to $1 \%$ BBP. As we can see, ICXT has a more remarkable effect in the US26 continental backbone network, being necessary to employ more robust modulation formats, even PM-BSPK in some cases. In MCF-enabled scenarios the most utilized modulation format is PM-QPSK, whilst for those equivalent MF solutions the most utilized one is PM16QAM. As for the case of the DT12 national backbone network, the shorter distances allow using higher order modulation formats. In the MCF-enabled scenario the modulation format used with the lowest efficiency is PM-QPSK, whilst in the equivalent MF scenario it is only necessary to lower it down to PM-16QAM. In addition, whereas in MCF-enabled scenarios the most utilized modulation format is 16-QAM, for MF ones the most utilized one is PM-64QAM. 


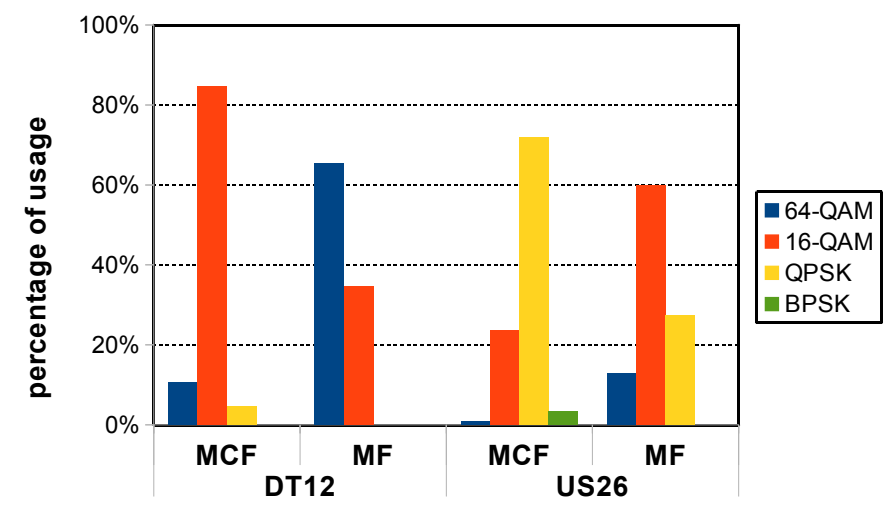

Fig. 8. Distribution of the usage of modulation formats in each network, DT12 and US26, for MCFenabled and equivalent MF scenarios. An offered load leading to $1 \% \mathrm{BBP}$ is assumed.

To end this subsection, we also compare the requirements of both MCF-enabled and equivalent MF solutions in terms of active transceivers in the network $(\alpha)$, extending the results obtained in the previous subsection. An increased number of active transceivers may be expected in the MF scenario, resulting from situation ii) analyzed before, as well as from the fact that the MF solution can accommodate more demands (an increased offered load can be supported for the same target BBP, e.g., 1\%, as seen in Fig. 8). On the other hand, situation iii) has the opposite effect; however, this is less likely to occur.

Fig. 9 illustrates the $\alpha$ CDFs for MCF-enabled and equivalent MF scenarios in the DT12 and US26 backbone networks, always employing the proposed PCA strategy. Again, an offered load leading to $1 \%$ BBP is assumed. As observed, our expectations are accomplished in both networks, being the $\alpha$ differences between MCF-enabled and equivalent MF scenarios more remarkable in the DT12 network $(12 \%$ and $9 \%$ increment in the peak number of active transceivers for MF vs. MCF, for DT12 and US26 networks, respectively). It shall be mentioned, though, that the estimated mean operational baud-rate of the transceivers in the MF scenarios is lower than in the MCF-enabled ones, i.e., 8.66 vs. 10.95 GBaud ( $26 \%$ increment), and 12.15 versus 14.69 GBaud (20\% increment), in DT12 and US26, respectively.

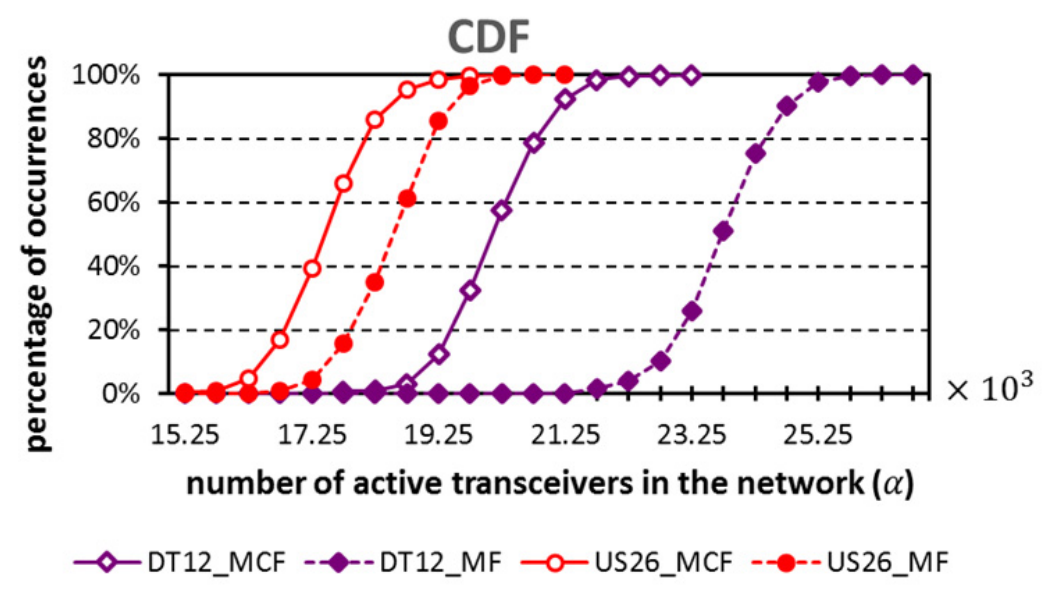

Fig. 9. $\alpha$ CDFs in DT12 and US26 networks for MCF-enabled and the equivalent MF solution. 


\section{CONCLUSIONS AND FUTURE WORK}

In this work, we provide a novel strategy to configure the spatial super-channels using MCFs and the joint-switching concept. Our proposal relies on the optimization of the number of cores employed to configure the Spa-SCh. The results show that for some specific demand bitrates employing all cores of the MCF (FCA strategy) is unnecessary, given the $12.5 \mathrm{GHz}$ spectral grid plus the required guard-band. Therefore, it is better to allocate an adequate number of cores (PCA strategy) for a better spectral occupancy. The adjustment of the employed cores per Spa-SCh contributes to network costs reduction, since it implies using a lower number of transceivers per node.

We evaluated the MCF solution based on BBP and the number of transceivers to be equipped network-wide and per node. The results show that, up to $44 \%$ of transceivers reduction can be obtained when PCA is considered instead of the FCA strategy, while maintaining the same network BBP performance. Moreover, although the considered 22-core MCFs introduce low ICXT figures, its impact can be significant depending on the network size and the guard-band width. Therefore, the ICXT value is a key element in the MCF design in order to provide an acceptable network performance in contrast with equivalent MF solutions.

PCA strategy allows releasing spatial and spectral resources with respect to FCA, which could not be assigned by default to other demands when joint-switching technique is applied. This penalty may be counteracted by using co-routing of demands, as described in reference [12], which deserves further investigation and is left for future work. Additionally, it would be interesting to evaluate our strategy with higher traffic profiles and/or using other SDM switching strategies, like independent-switching or fractional joint-switching [19], which provide elasticity to the space dimension. Finally, future work could also be focused on the evaluation of the MCFs with less core count and/or less ICXT to compare the performance results with the same traffic profile used in this work. There may be an optimum number of cores for the MCFs to be used for every traffic profile and network size.

\section{ACKNOWLEDGEMENTS}

This work has been partially funded by the Spanish National Project SUNSET (TEC2014-59583C2-1-R), which receives funding from FEDER.

Rubén Rumipamba is recipient of a full scholarship from Secretaría Nacional de Ciencia y Tecnología (SENESCYT) - Ecuador.

\section{REFERENCES}

[1] P. J. Winzer, "Spatial Multiplexing : The Next Frontier in Network Capacity Scaling," Eur. Conf. Exhib. Opt. Commun., p. We.1.D.1, 2013.

[2] D. J. Richardson, J. M. Fini, and L. E. Nelson, "Space Division Multiplexing in Optical Fibres," Nat. Photonics, vol. 7, pp. 354-362, 2013.

[3] J. Sakaguchi, Y. Awaji, N. Wada, A. Kanno, T. Kawanishi, T. Hayashi, T. Taru, T. Kobayashi, and M. Watanabe, "Space division multiplexed transmission of 109-Tb/s data signals using homogeneous seven-core fiber," J. Light. Technol., vol. 30, no. 4, pp. 658-665, 2012. 
[4] A. Sano, H. Takara, T. Kobayashi, H. Kawakami, H. Kishikawa, T. Nakagawa, Y. Miyamoto, Y. Abe, H. Ono, K. Shikama, M. Nagatani, T. Mori, Y. Sasaki, I. Ishida, K. Takenaga, S. Matsuo, K. Saitoh, M. Koshiba, M. Yamada, H. Masuda, and T. Morioka, "409-Tb/s + 409-Tb/s crosstalk suppressed bidirectional MCF transmission over $450 \mathrm{~km}$ using propagation-direction interleaving," vol. 21, no. 14, pp. 16777-16783, 2013.

[5] J. Sakaguchi, W. Klaus, B. J. Puttnam, J. M. D. Mendinueta, Y. Awaji, N. Wada, Y. Tsuchida, K. Maeda, M. Tadakuma, K. Imamura, R. Sugizaki, T. Kobayashi, Y. Tottori, M. Watanabe, and R. V Jensen, "19-core MCF transmission system using EDFA with shared core pumping coupled via free-space optics.," Opt. Express, vol. 22, no. 1, pp. 90-5, 2014.

[6] S. Matsuo, K. Takenaga, Y. Sasaki, Y. Amma, S. Saito, K. Saitoh, T. Matsui, K. Nakajima, T. Mizuno, H. Takara, Y. Miyamoto, and T. Morioka, "High-Spatial-Multiplicity Multicore Fibers for Future Dense Space-Division-Multiplexing Systems," J. Light. Technol., vol. 34, no. 6, pp. 1464-1475, Mar. 2016.

[7] International Telecommunication Union - ITU-T, "G.694.1 (02/2012), Spectral grids for WDM applications: DWDM frequency grid," Ser. G.694.1, pp. 1-16, 2012.

[8] D. Klonidis, F. Cugini, O. Gerstel, M. Jinno, V. Lopez, E. Palkopoulou, M. Sekiya, D. Siracusa, G. Thou??non, and C. Betoule, "Spectrally and spatially flexible optical network planning and operations," IEEE Commun. Mag., vol. 53, no. 2, pp. 69-78, 2015.

[9] P. Sayyad Khodasenas, J.-M. Rivas-Moscoso, D. Siracusa, F. Pederzolli, B. Shariati, D. Klonidis, E. Salvadori, and I. Tomkos, "Comparison of Spectral and Spatial Super-channel Allocation Schemes for SDM Networks," J. Light. Technol., vol. 8724, no. c, pp. 1-1, 2016.

[10] P. J. Winzer, "High-spectral-efficiency optical modulation formats," J. Light. Technol., vol. 30, no. 24, pp. 3824-3835, 2012.

[11] L. E. Nelson, M. D. Feuer, K. Abedin, X. Zhou, T. F. Taunay, J. M. Fini, B. Zhu, R. Isaac, R. Harel, G. Cohen, and D. M. Marom, "Spatial superchannel routing in a two-span ROADM system for space division multiplexing," J. Light. Technol., vol. 32, no. 4, pp. 783-789, 2014.

[12] F. Pederzolli, D. Siracusa, J. M. Rivas, B. Shariati, E. Salvadori, and I. Tomkos, "Spatial Group Sharing for SDM Optical Networks with Joint Switching," 20th Int. Conf. Opt. Netw. Des. Model., no. Ondm, pp. 182-187, 2016.

[13] B. J. Puttnam, R. S. Luis, J. Sakaguchi, W. Klaus, J. M. D. Mendinueta, Y. Awaji, and N. Wada, "High capacity multi-core fiber systems," 2016, pp. 29-34.

[14] P. Poggiolini, G. Bosco, A. Carena, V. Curri, Y. Jiang, and F. Forghieri, "The GN-Model of Fiber Non-Linear Propagation and its Applications," J. Light. Technol., vol. 32, no. 4, pp. 694-721, Feb. 2014.

[15] A. Carena, G. Bosco, V. Curri, Y. Jiang, P. Poggiolini, and F. Forghieri, "EGN model of non-linear fiber propagation," Opt. Express, vol. 22, no. 13, p. 16335, Jun. 2014.

[16] P. J. Winzer, a. H. Gnauck, a. Konczykowska, F. Jorge, and J.-Y. Dupuy, "Penalties from in-band crosstalk for advanced optical modulation formats," 2011 37th Eur. Conf. Exhib. Opt. Commun., no. 1, pp. 1-3, 2011.

[17] O. Gerstel, M. Jinno, A. Lord, and S. J. Ben Yoo, "Elastic optical networking: A new dawn for the optical layer?," IEEE Commun. Mag., vol. 50, no. 2, pp. 12-20, 2012.

[18] N. Goldshtein, D. Sinefeld, O. Ori, L. Pascar, L. Pascar, R. Zektzer, and D. M. Marom, 
"Fine Resolution Photonic Spectral Processor Using a Waveguide Grating Router With Permanent Phase Trimming," J. Light. Technol., vol. 34, no. 2, pp. 379-385, Jan. 2016.

[19] D. M. Marom and M. Blau, "Switching solutions for WDM-SDM optical networks," IEEE Commun. Mag., vol. 53, no. 2, pp. 60-68, 2015. 